\title{
One-prong $\tau$ decays with neutral kaons
}

\section{L3 Collaboration}

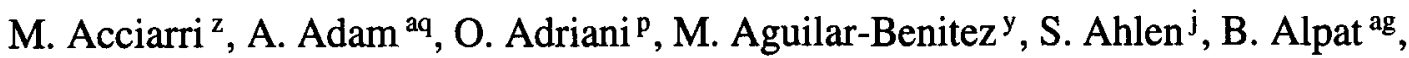

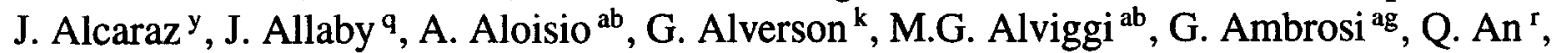
H. Anderhub ${ }^{\text {at }}$, V.P. Andreev ${ }^{\text {ak }}$, T. Angelescu ${ }^{\ell}$, D. Antreasyan ${ }^{\text {h }}$, A. Arefiev ${ }^{\text {aa }}$, T. Azemoon ${ }^{c}$, T. Aziz ${ }^{i}$, P.V.K.S. Baba ${ }^{\mathrm{r}}$, P. Bagnaia ${ }^{\mathrm{aj}, \mathrm{q}}$, L. Baksay ${ }^{\text {ap }}$, R.C. Ball ${ }^{\mathrm{c}}$, S. Banerjee ${ }^{\mathrm{i}}, \mathrm{K}$. Banicz $^{\mathrm{aq}}$, R. Barillère ${ }^{\mathrm{q}}$, L. Barone ${ }^{\mathrm{aj}}$, P. Bartalini ${ }^{\mathrm{ag}}, \mathrm{A}$. Baschirotto ${ }^{\mathrm{z}}$, M. Basile $^{\mathrm{h}}$, R. Battiston ${ }^{\mathrm{ag}}$, A. Bay $^{\mathrm{v}}$, F. Becattini ${ }^{p}$, U. Becker ${ }^{\circ}$, F. Behner ${ }^{\text {at }}$, Gy.L. Bencze ${ }^{m}$, J. Berdugo $^{y}$, P. Berges $^{\circ}$, B. Bertucci ${ }^{q}$, B.L. Betev ${ }^{\text {at }}$, M. Biasini ${ }^{\text {ag }}$, A. Biland ${ }^{\text {at }}$, G.M. Bilei ${ }^{\text {ag }}$, R. Bizzarri ${ }^{\text {aj }}$, J.J. Blaising ${ }^{q}$,

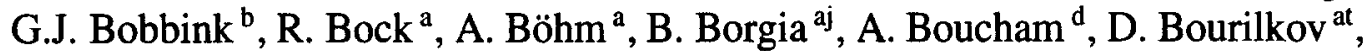
M. Bourquin ${ }^{\text {s, D. Boutigny }}{ }^{d}$, B. Bouwens ${ }^{\text {b }}$, E. Brambilla ${ }^{\circ}$, J.G. Branson ${ }^{a}{ }$, V. Brigljevic ${ }^{\text {at }}$, I.C. Brock $^{\text {ah }}$, A. Bujak ${ }^{\text {aq }}$, J.D. Burger ${ }^{\text {, }}$, W.J. Burger ${ }^{\text {s }}$, C. Burgos ${ }^{\mathrm{y}}$, J. Busenitz ${ }^{\text {ap }}$, A. Buytenhuijs ${ }^{\text {ad }}$, X.D. Cai ${ }^{\mathrm{r}}$, M. Capell ${ }^{\text {}}$, G. Cara Romeo ${ }^{\mathrm{h}}$, M. Caria ${ }^{\mathrm{ag}}$, G. Carlino $^{\mathrm{ab}}$,

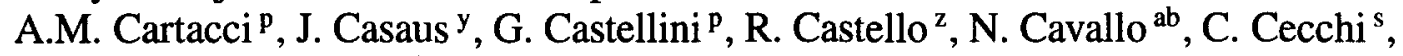
M. Cerrada ${ }^{y}$, F. Cesaroni ${ }^{\text {aj }}$, M. Chamizo ${ }^{y}$, A. Chan ${ }^{\text {av }}$, Y.H. Chang ${ }^{\text {av }}$, U.K. Chaturvedi ${ }^{r}$, M. Chemarin ${ }^{x}$, A. Chen ${ }^{\text {av }}$, C. Chen ${ }^{f}$, G. Chen ${ }^{f}$, G.M. Chen ${ }^{f}$, H.F. Chen ${ }^{t}$, H.S. Chen ${ }^{f}$,

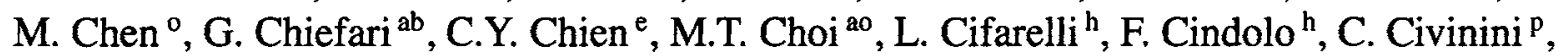

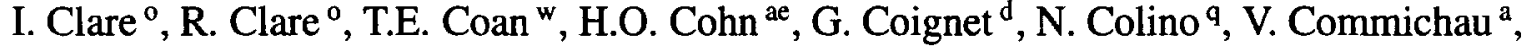
S. Costantini ${ }^{\text {aj }}$, F. Cotorobai ${ }^{\ell}$, B. de la Cruz ${ }^{y}$, X.T. Cui ${ }^{r}$, X.Y. Cui ${ }^{r}$, T.S. Dai ${ }^{o}$, R. D'Alessandro ${ }^{p}$, R. de Asmundis ${ }^{\text {ab }}$, H. De Boeck ${ }^{\text {ad }}$, A. Degré ${ }^{d}$, K. Deiters ${ }^{\text {ar }}$, E. Dénes ${ }^{\mathrm{m}}$, P. Denes ${ }^{\text {a }}$, F. DeNotaristefani ${ }^{\text {aj }}$, D. DiBitonto ${ }^{\text {ap }}$, M. Diemoz ${ }^{\text {aj }}$, C. Dionisi ${ }^{\text {aj }}$, M. Dittmar ${ }^{\text {at }}$,

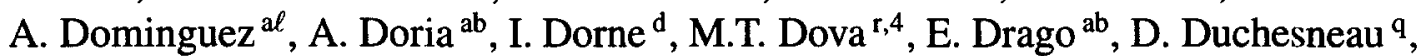
P. Duinker ${ }^{\text {b }}$, I. Duran ${ }^{\text {am }}$, S. Dutta ${ }^{i}$, S. Easo ${ }^{\text {ag }}$, Yu. Efremenko ${ }^{\text {ae }}$, H. El Mamouni ${ }^{x}$, A. Engler ${ }^{\text {ah }}$, F.J. Eppling ${ }^{\circ}$, F.C. Erné ${ }^{b}$, J.P. Ernenwein ${ }^{x}$, P. Extermann ${ }^{\text {s }}$, R. Fabbretti ${ }^{\text {ar }}$, M. Fabre ${ }^{\text {ar }}$,

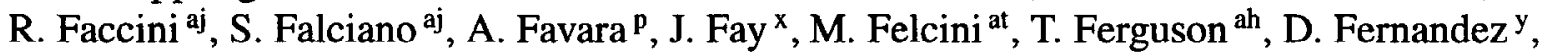
G. Fernandez ${ }^{y}$, F. Ferroni ${ }^{\text {aj }}$, H. Fesefeldt ${ }^{a}$, E. Fiandrini ${ }^{\text {ag }}$, J.H. Field ${ }^{\mathrm{s}}$, F. Filthaut ${ }^{\text {ah }}$, P.H. Fisher ${ }^{\circ}$, G. Forconi ${ }^{\circ}$, L. Fredj ${ }^{\mathrm{s}}$, K. Freudenreich ${ }^{\text {at }}$, M. Gailloud ${ }^{\mathrm{v}}$, Yu. Galaktionov ${ }^{\text {aa,o, }}$, S.N. Ganguli i , P. Garcia-Abia ${ }^{y}$, S.S. Gau ${ }^{\text {k }}$, S. Gentile ${ }^{\text {aj }}$, J. Gerald ${ }^{\mathrm{e}}$, N. Gheordanescu ${ }^{\ell}$,

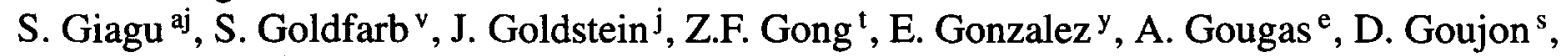
G. Gratta ${ }^{\text {af }}$, M.W. Gruenewald ${ }^{\mathrm{g}}$, C. Gu ${ }^{\mathrm{r}}$, M. Guanziroli ${ }^{\mathrm{r}}$, V.K. Gupta ${ }^{\text {ai }}$, A. Gurtu ${ }^{\mathrm{i}}$, H.R. Gustafson ${ }^{\text {c }}$, L.J. Gutay ${ }^{\text {aq }}$, B. Hartmann ${ }^{a}$, A. Hasan ${ }^{\text {ac }}$, J.T. He ${ }^{\text {f }}$, T. Hebbeker ${ }^{\text {g }}$, A. Hervé ,

K. Hilgers ${ }^{\text {a }}$, W.C. van Hoek ${ }^{\text {ad }}$, H. Hofer ${ }^{\text {at }}$, H. Hoorani ${ }^{\text {s }, ~ S . R . ~ H o u ~}{ }^{\text {av }}$, G. Hu ${ }^{r}$, M.M. Ilyas ${ }^{r}$, 
V. Innocente ${ }^{q}$, H. Janssen ${ }^{d}$, B.N. Jin ${ }^{f}$, L.W. Jones ${ }^{c}$, P. de Jong ${ }^{o}$, I. Josa-Mutuberria ${ }^{y}$, A. Kasser ${ }^{v}$, R.A. Khan ${ }^{\text {r }}$, Yu. Kamyshkov ${ }^{\text {ae }}$, P. Kapinos ${ }^{\text {as }}$, J.S. Kapustinsky ${ }^{\text {w}}$, Y. Karyotakis ${ }^{d}$, M. Kaur ${ }^{\mathrm{r}}$, S. Khokhar ${ }^{\mathrm{r}}$, M.N. Kienzle-Focacci ${ }^{\mathrm{s}}$, D. Kim ${ }^{\mathrm{e}}$, J.K. Kim ${ }^{\text {ao }}$, S.C. Kim ${ }^{\text {ao }}$, Y.G. Kim ${ }^{\text {ao }}$, W.W. Kinnison ${ }^{\text {w }}$, A. Kirkby ${ }^{\text {af }}$, D. Kirkby ${ }^{\text {af }}$, J. Kirkby ${ }^{\text {q }}$, S. Kirsch ${ }^{\text {as }}$, W. Kittel ${ }^{\text {ad }}$, A. Klimentov ${ }^{\text {,aa }}$, A.C. König ad , E. Koffeman ${ }^{\text {b }}$, O. Kornadt ${ }^{\text {a }}$, V. Koutsenko ${ }^{\text {o,aa, }}$, A. Koulbardis ${ }^{\text {ak }}$, R.W. Kraemer ${ }^{\text {ah }}$, T. Kramer ${ }^{\circ}$, W. Krenz ${ }^{\text {a }}$, H. Kuijten ${ }^{\text {ad }}$, A. Kunin ${ }^{0 . a a}$, P. Ladron de Guevara ${ }^{y}$, G. Landi ${ }^{p}$, C. Lapoint ${ }^{\mathrm{o}}$, K. Lassila-Perini ${ }^{\text {at }}$, P. Laurikainen ${ }^{\mathrm{u}}$, M. Lebeau ${ }^{\text {q }}$, A. Lebedev ${ }^{\text {}}$, P. Lebrun ${ }^{\text {X }}$, P. Lecomte ${ }^{\text {at }}$, J. Lccoq ${ }^{\text {d }}$, P. Lecoq ${ }^{\text {q }}$, P. Le Coultre ${ }^{\text {at }}$, J.S. Lee ${ }^{\text {ao }}$, K.Y. Lee ${ }^{\text {ao }}$, C. Leggett ${ }^{\text {c }}$, J.M. Le Goff ${ }^{q}$, R. Leiste ${ }^{\text {as }}$, M. Lenti ${ }^{\text {p }}$, E. Leonardi ${ }^{\text {aj }}$, P. Levtchenko ak ${ }^{\text {, C. } \text { Li }^{\text {tr. }}, \text { E. Lieb }}{ }^{\text {as }}$, W.T. Lin ${ }^{\text {av }}$, F.L. Linde ${ }^{\text {b }}$, B. Lindemann ${ }^{\text {a }}$, L. Lista ${ }^{\text {ab }}$, Y. Liu ${ }^{r}$, Z.A. Liu ${ }^{f}$, W. Lohmann ${ }^{\text {as }}$, E. Longo ${ }^{\text {aj }}$, W. Lu ${ }^{\text {af }}$, Y.S. Lu ${ }^{\text {f }}$, K. Lübelsmeyer ${ }^{\text {a }}$, C. Luci ${ }^{\text {aj }}$, D. Luckey ${ }^{\circ}$, L. Ludovici ${ }^{\text {aj }}$, L. Luminari ${ }^{\text {aj }}$, W. Lustermann ${ }^{\text {ar }}$, W.G. Ma ${ }^{t}$, A. Macchiolo ${ }^{p}$, M. Maity ${ }^{\mathrm{i}}$, L. Malgeri ${ }^{\text {aj }}$, R. Malik ${ }^{\mathrm{r}}$, A. Malinin ${ }^{\text {aa }}$, C. Maña ${ }^{\mathrm{y}}$, S. Mangla ${ }^{\mathrm{i}}$, M. Maolinbay $^{\text {at }}$, P. Marchesini at, A. Marin ${ }^{\text {j, J.P. Martin }}{ }^{\text {x }}$, F. Marzano ${ }^{\text {aj }}$, G.G.G. Massaro ${ }^{\text {b }, ~ K . ~ M a z u m d a r ~}{ }^{i}$, D. McNally ${ }^{\mathrm{q}}$, S. Mele ${ }^{\mathrm{ab}}$, M. Merk ${ }^{\text {ah }}$, L. Merola $^{\text {ab }}$, M. Meschini ${ }^{\text {p }}$, W.J. Metzger ${ }^{\text {ad }}$, Y. Mi $^{\text {v }}$, A. Mihul ${ }^{\ell}$, A.J.W. van Mil ad, Y. Mir ${ }^{\mathrm{r}}$, G. Mirabelli ${ }^{\text {aj }}$, J. Mnich ${ }^{\mathrm{q}}$, M. Möller ${ }^{\mathrm{a}}$, V. Monaco ${ }^{\text {aj, }}$ B. Monteleoni ${ }^{\mathrm{p}}$, R. Moore ${ }^{\mathrm{c}}$, R. Morand ${ }^{\mathrm{d}}$, S. Morganti ${ }^{\text {aj }}$, N.E. Moulai ${ }^{\mathrm{r}}$, R. Mount ${ }^{\text {af }}$, S. Müller ${ }^{\text {a }}$, E. Nagy ${ }^{\mathrm{m}}$, S. Nahn ${ }^{\mathrm{o}}$, M. Napolitano ${ }^{\text {ab }}$, F. Nessi-Tedaldi ${ }^{\text {at }}, \mathrm{H}$. Newman ${ }^{\text {af }}$, M.A. Niaz ${ }^{\text {r }}$ A. Nippe ${ }^{\text {a }}$, H. Nowak ${ }^{\text {as }}$, G. Organtini ${ }^{\text {aj }}$, R. Ostonen ${ }^{\text {u, D. Pandoulas }}{ }^{\text {a }}$,

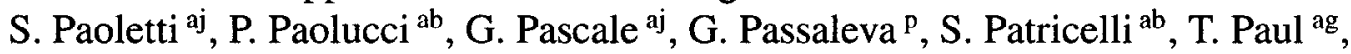
M. Pauluzzi ${ }^{\text {ag }}$, C. Paus ${ }^{\text {a }}$, F. Pauss ${ }^{\text {at }}$, Y.J. Pei ${ }^{\text {a }}$, S. Pensotti ${ }^{\text {z }}$, D. Perret-Gallix ${ }^{\text {d }}$, A. Pevsner ${ }^{\text {e }}$, D. Piccolo ${ }^{\text {ab }}$, M. Pieri ${ }^{\text {p }}$, J.C. Pinto ${ }^{\text {ah }}$, P.A. Piroué ai ${ }^{\text {a E. Pistolesi }}{ }^{\text {P }}$, V. Plyaskin ${ }^{\text {aa }}$, M. Pohl ${ }^{\text {at }}$, V. Pojidaev aa,p , H. Postema ${ }^{\circ}$, N. Produit ${ }^{\mathrm{s}}$, K.N. Qureshi ${ }^{\mathrm{r}}$, R. Raghavan ${ }^{\mathrm{i}}$, G. Rahal-Callot ${ }^{\text {at }}$, P.G. Rancoita ${ }^{z}$, M. Rattaggi ${ }^{z}$, G. Raven ${ }^{\text {b }}$, P. Razis ${ }^{\text {ac }}$, K. Read ${ }^{\text {ae }}$, M. Redaelli ${ }^{\text {}}$, D. Ren ${ }^{\text {at }}$, Z. Ren ${ }^{\mathrm{r}}$, M. Rescigno ${ }^{\text {aj }}$, S. Reucroft ${ }^{\mathrm{k}}$, A. Ricker ${ }^{\mathrm{a}}$, S. Riemann ${ }^{\text {as }}$, B.C. Riemers ${ }^{\mathrm{aq}}, \mathrm{K}$. Riles ${ }^{\mathrm{c}}$, O. Rind ${ }^{c}$, H.A. Rizvi ${ }^{r}$, S. Ro ${ }^{a 0}$, A. Robohm ${ }^{\text {at }}$, J. Rodin ${ }^{\circ}$, F.J. Rodriguez ${ }^{y}$, B.P. Roe ${ }^{c}$, M. Röhner ${ }^{a}$, S. Röhner ${ }^{a}$, L. Romero ${ }^{y}$, S. Rosier-Lees ${ }^{d}$, Ph. Rosselet ${ }^{v}$, W. van Rossum ${ }^{b}$, S. Roth ${ }^{\text {a }}$, J.A. Rubio ${ }^{\text {, }}$ H. Rykaczewski ${ }^{\text {at }}$, J. Salicio ${ }^{q}$, J.M. Salicio ${ }^{y}$, E. Sanchez ${ }^{y}$, A. Santocchia ${ }^{\text {ag }}$, M.E. Sarakinos ${ }^{\text {u }}$, S. Sarkar ${ }^{i}$, G. Sartorelli ${ }^{\text {r }}$, M. Sassowsky ${ }^{a}$, G. Sauvage ${ }^{d}$, C. Schäfer ${ }^{a}$, V. Schegelsky ${ }^{\text {ak }}$, D. Schmitz ${ }^{a}$, P. Schmitz ${ }^{\text {a }}$, M. Schneegans ${ }^{\text {d }}$, B. Schoeneich ${ }^{\text {as }}$, N. Scholz ${ }^{\text {at }}$, H. Schopper au, D.J. Schotanus ${ }^{\text {ad }}$, R. Schulte ${ }^{\text {a }}$, K. Schultze ${ }^{\text {a }}$, J. Schwenke ${ }^{\text {a }}$, G. Schwering ${ }^{a}$, C. Sciacca ${ }^{\text {ab }}$, R. Sehgal ${ }^{\text {r }}$, P.G. Seiler ${ }^{\text {ar }}$, J.C. Sens ${ }^{\text {av }}$, L. Servoli ${ }^{\text {ag, }}$, S. Shevchenko ${ }^{\text {af }}$, N. Shivarov an, V. Shoutko ${ }^{\text {aa }}$, J. Shukla ${ }^{\text {w }}$, E. Shumilov ${ }^{\text {aa }}$, D. Son ${ }^{\text {ao }}$, A. Sopczak ${ }^{q}$, V. Soulimov ${ }^{\text {ab }}$, B. Smith ${ }^{\circ}$, T. Spickermann $^{\text {a }}$, P. Spillantini ${ }^{\mathrm{p}}$, M. Steuer ${ }^{\text {, }}$, D.P. Stickland ${ }^{\text {ai }}$, F. Sticozzi ${ }^{\circ}$, H. Stone ${ }^{\text {ai }}$, B. Stoyanov an ${ }^{\text {an }}$ K. Strauch ${ }^{n}$, K. Sudhakar ${ }^{i}$, G. Sultanov ${ }^{\mathrm{r}}$, L.Z. Sun ${ }^{\mathrm{t}, \mathrm{r}}$, G.F. Susinno ${ }^{\mathrm{s}}$, H. Suter ${ }^{\text {at }}$, J.D. Swain ${ }^{\mathrm{r}}$, A.A. Syed ${ }^{\text {ad }}$, X.W. Tang ${ }^{\mathrm{f}}$, L. Taylor ${ }^{\mathrm{k}}$, R. Timellini ${ }^{\mathrm{h}}$, Samuel C.C. Ting ${ }^{\mathrm{o}}$, S.M. Ting ${ }^{\mathrm{o}}$, O. Toker ${ }^{\mathrm{ag}}$, M. Tonutti ${ }^{\mathrm{a}}$, S.C. Tonwar ${ }^{\mathrm{i}}, \mathrm{J}$. Tóth ${ }^{\mathrm{m}}$, A. Tsaregorodtsev ${ }^{\mathrm{ak}}, \mathrm{G}$. Tsipolitis ah ${ }^{\text {ah }}$ C. Tully ai , H. Tuchscherer ${ }^{\text {ap, }}$, J. Ulbricht ${ }^{\text {at }}$, L. Urbán ${ }^{\mathrm{m}}$, U. Uwer ${ }^{\mathrm{a}}$, E. Valente ${ }^{\text {aj }}$, R.T. Van de Walle ${ }^{\text {ad }}$, I. Vetlitsky ${ }^{\text {aa }}$, G. Viertel ${ }^{\text {at }}$, P. Vikas ${ }^{\mathrm{r}}$, U. Vikas ${ }^{\mathrm{r}}, \mathrm{M}$. Vivargent ${ }^{\mathrm{d}}, \mathrm{R}$. Voelkert ${ }^{\text {as }}, \mathrm{H}$. Vogel ${ }^{\text {ah }}, \mathrm{H}$. Vogt ${ }^{\text {as }}$, 
I. Vorobiev ${ }^{\text {aa }}$, A.A. Vorobyov ${ }^{\text {ak }}$, An.A. Vorobyov ${ }^{\text {ak }}$, L. Vuilleumier ${ }^{\mathrm{v}}$, M. Wadhwa ${ }^{\mathrm{y}}$, W. Wallraff ${ }^{\mathrm{a}}$, J.C. Wang ${ }^{\circ}$, X.L. Wang ${ }^{\text {}}$, Y.F. Wang ${ }^{n}$, Z.M. Wang ${ }^{\text {r,t }}$, A. Weber ${ }^{\mathrm{a}}$, R. Weill ${ }^{\mathrm{v}}$, C. Willmott ${ }^{y}$, F. Wittgenstein ${ }^{\mathrm{q}}$, S.X. Wu ${ }^{\mathrm{r}}$, S. Wynhoff ${ }^{\mathrm{a}}$, J. Xu ${ }^{\mathrm{j}}$, Z.Z. Xu ${ }^{\mathrm{t}}$, B.Z. Yang ${ }^{\mathrm{t}}$, C.G. Yang ${ }^{f}$, G. Yang ${ }^{\mathrm{r}}$, X.Y. Yao ${ }^{\mathrm{f}}$, C.H. Ye ${ }^{\mathrm{r}}$, J.B. Ye ${ }^{\text {', Q }}$ Q. Ye ${ }^{\mathrm{r}}$, S.C. Yeh ${ }^{\text {av }}$, J.M. You ${ }^{\text {ah }}$, N. Yunus ${ }^{\mathrm{r}}$, M. Yzerman ${ }^{\mathrm{b}}$, C. Zaccardelli af ${ }^{\text {af }}$ An. Zalite ${ }^{\mathrm{ak}}$, P. Zemp ${ }^{\text {at }}$, J.Y. Zeng ${ }^{\mathrm{f}}$, M. Zeng ${ }^{\mathrm{r}}$,

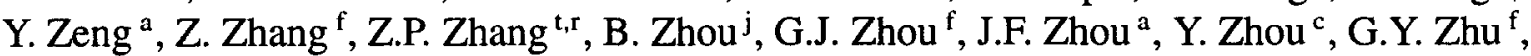
R.Y. Zhu ${ }^{\text {af }}$, A. Zichichi ${ }^{\text {h,q,r, }}$, B.C.C. van der Zwaan ${ }^{\text {b }}$

'1. Physikalisches Institut, RWTH, D-52056 Aachen, FRG ${ }^{1}$

III. Physikalisches Institut, RWTH, D-52056 Aachen, FRG ${ }^{1}$

${ }^{b}$ National Institute for High Energy Physics, NIKHEF, NL-1009 DB Amsterdam, The Netherlands

c University of Michigan, Ann Arbor, MI 48109, USA

¿ Laboratoire d'Annecy-le-Vieux de Physique des Particules, LAPP,IN2P3-CNRS, BP 110, F-7494I Annecy-le-Vieux CEDEX, France

¿ Johns Hopkins University, Baltimore, MD 21218, USA

'Institute of High Energy Physics, IHEP, 100039 Beijing, China

g Humboldt University, D-10099 Berlin, FRG ${ }^{1}$

h INFN-Sezione di Bologna, I-40126 Bologna, Italy

i Tata Institute of Fundamental Research, Bombay 400005 , India

i Boston University, Boston, MA 02215, USA

${ }^{k}$ Northeastern University, Boston, MA 02115, USA

${ }^{\ell}$ Institute of Atomic Physics and University of Bucharest, R-76900 Bucharest, Romania

m Central Research Institute for Physics of the Hungarian Academy of Sciences, H-1525 Budapest 114, Hungary ${ }^{2}$

" Harvard University, Cambridge, MA 02139, USA

- Massachusetts Institute of Technology, Cambridge, MA 02139, USA

'INFN Sezione di Firenze and University of Florence, I-50125 Florence, Italy

${ }^{4}$ European Laboratory for Particle Physics, CERN, CH-1211 Geneva 23, Switzerland

r World Laboratory, FBLJA Project, CH-1211 Geneva 23, Switzerland

s University of Geneva, CH-1211 Geneva 4, Switzerland

${ }^{t}$ Chinese University of Science and Technology, USTC, Hefei, Anhui 230 029, China

' SEFT, Research Institute for High Energy Physics, P.O. Box 9, SF-00014 Helsinki, Finland

$\checkmark$ University of Lausanne, CH-IOIS Lausanne, Switzerland

w Los Alamos National Laboratory, Los Alamos, NM 87544, USA

x Institut de Physique Nucléaire de Lyon. IN2P3-CNRS.Université Claude Bernard. F-69622 Villeurbanne Cedex. France

y Centro de Investigaciones Energeticas, Medioambientales y Tecnologicas, CIEMAT, E-28040 Madrid, Spain ${ }^{3}$

${ }^{2}$ INFN-Sezione di Milano, 1-20133 Milan, Italy

aa Institute of Theoretical and Experimental Physics, ITEP, Moscow, Russia

ab INFN-Sezione di Napoli and University of Naples, I-80125 Naples, Italy

ac Department of Natural Sciences, University of Cyprus, Nicosia, Cyprus

ad University of Nymegen and NIKHEF, NL-6525 ED Nymegen, The Netherlands

ae Oak Ridge National Laboratory, Oak Ridge, TN 37831, USA

af California Institute of Technology, Pasadena, CA 91125, USA

ag INFN-Sezione di Perugia and Universitá Degli Studi di Perugia, 1-06100 Perugia, Italy

ah Carnegie Mellon University, Pittsburgh, PA 15213, USA

ai Princeton University, Princeton, NJ 08544, USA

aj INFN-Sezione di Roma and University of Rome. "La Sapienza", I-00185 Rome, Italy

ak Nuclear Physics Institute, St. Petersburg, Russia

al University of California, San Diego, CA 92093, USA

am Dept. de Fisica de Particulas Elementales, Univ. de Santiago, E-15706 Santiago de Compostela, Spain

an Bulgarian Academy of Sciences, Central Laboratory of Mechatronics and Instrumentation, BU-1113 Sofia, Bulgaria

ao Center for High Energy Physics, Korea Advanced Inst. of Sciences and Technology, 305-701 Taejon, South Korea

ap University of Alabama, Tuscaloosa, AL 35486, USA

aq Purdue University, West Lafayette, IN 47907, USA

ar Paul Scherrer Institut, PSI, CH-5232 Villigen, Switzerland

as DESY-Institut für Hochenergiephysik, D-15738 Zeuthen, FRG 
at Eidgenössische Technische Hochschule, ETH Zürich, CH-8093 Zürich, Switzerland

au University of Hamburg, D-22761 Hamburg, FRG

av High Energy Physics Group, Taiwan, ROC

Received 4 April 1995

Editor: K. Winter

\begin{abstract}
We have analyzed one-prong $\tau$ decays with neutral kaons using the information from a fine-grained hadron calorimeter. The data sample consists of $43500 \mathrm{Z} \rightarrow \tau^{+} \tau^{-}(\gamma)$ events collected by the L3 detector at LEP in 1991, 1992 and 1993. The following branching fractions are measured: $\mathcal{B}\left(\tau^{-} \rightarrow \nu_{\tau} \pi^{-} \overline{\mathbf{K}}^{0}\right)=0.0095 \pm 0.0015$ (stat) \pm 0.0006 (syst); $\mathcal{B}\left(\tau^{-} \rightarrow\right.$ $\left.\nu_{\tau} \pi^{-} \pi^{0} \overline{\mathrm{K}}^{0}\right)=0.0041 \pm 0.0012$ (stat) \pm 0.0003 (syst) and $\mathcal{B}\left(\tau^{-} \rightarrow \nu_{r} \pi^{-} \mathbf{K}^{0} \overline{\mathrm{K}}^{0}\right)=0.0031 \pm 0.0012$ (stat) \pm 0.0004 (syst)
\end{abstract}

\section{Introduction}

Measurements of the branching fractions of $\tau$ lepton decays with neutral kaons are important for understanding $\tau$ decays [1], in particular the compatibility of inclusive and exclusive branching fractions. They also provide new information on the dynamics of neutral kaon production which is not yet well understood. Many factors affect the calculation of the branching fractions for these decays, such as Cabibbo-suppression for modes with odd numbers of kaons, very limited phase space for the modes with two kaons, QCD anomalies and uncertainties in the resonant structure. Their interplay prevents firm theoretical predictions from being made $[2,3]$.

In this paper we present an analysis of the following decay modes: $\tau \rightarrow \nu_{\tau} \pi^{-} \overline{\mathbf{K}}^{0}, \tau^{-} \rightarrow \nu_{\tau} \pi^{-} \pi^{0} \overline{\mathbf{K}}^{0}$, and $\tau^{-} \rightarrow \nu_{\tau} \pi^{-} \mathbf{K}^{0} \overline{\mathbf{K}}^{0}$, where the charge conjugate decays are also implied here and throughout this paper. The fine-grained hadron calorimeter of L3 is used to detect neutral kaons and to measure their energies and directions. The selection of $\tau$ decays with neutral kaons is based on the coincidence of high energy deposition in the hadron calorimeter and a low momentum charged track detected in the central tracker. The L3 detector

\footnotetext{
' Supported by the German Bundesministerium für Bildung, Wissenschaft, Forschung und Technolugie.

${ }^{2}$ Supported by the Hungarian OTKA fund under contract number 2970.

${ }^{3}$ Supported also by the Comisión Interministerial de Ciencia y Technología.

${ }^{4}$ Also supported by CONICET and Universidad Nacional de La Plata, CC 67, 1900 La Plata, Argentina.
}

is well suited to this measurement as the efficiencies for detecting the $K_{L}$ and $K_{S}$ components of $K^{0}$ are comparable due to the small decay volume.

The data sample used in this analysis corresponds to an integrated luminosity of $69 \mathrm{pb}^{-1}$ collected at $\sqrt{s} \approx M_{\mathrm{Z}}$ during the 1991,1992 and 1993 LEP running periods.

\section{The $\mathrm{L3}$ detector}

The $\mathrm{L} 3$ detector is described in detail in Ref. [4]. The $\mathrm{e}^{+} \mathrm{e}^{-}$collision point is surrounded by a tracking chamber, a high resolution electromagnetic calorimeter, a cylindrical shell of scintillation counters, a hadron calorimeter, and a muon chamber system. The detector is installed in a large magnet providing a uniform 0.5 Tesla field.

The hadron calorimeter (HCAL) is the key subdetector for this measurement. It covers the polar angle range $5^{\circ}<\theta<175^{\circ}$ and consists of depleted uranium absorber plates interleaved with proportional wire chambers oriented alternately parallel and perpendicular to the beam direction. The readout, which is grouped into cells in both of these projections as well as in depth, provides a three dimensional pattern for hadronic showers. The energy resolution of the calorimeter for $\pi^{ \pm}$is determined to be $55 \% / \sqrt{E(\mathrm{GeV})}+8 \%$ in the central region. The angular resolution is better than $40 \mathrm{mrad}$ in both the polar and azimuthal projections for hadronic showers above $6 \mathrm{GeV}$, which is approximately the minimum energy of $\mathrm{K}^{0}$ 's from $\tau$ decays at LEP. The detailed 
study of the hadron calorimeter prototype is described elsewhere [5].

The electromagnetic calorimeter (ECAL) is composed of bismuth germanate crystals in the shape of truncated pyramids pointing to the interaction region. The ECAL barrel has a polar angle coverage of $42^{\circ}<\theta<138^{\circ}$, while the end-caps cover the regions $12^{\circ}<\theta<38^{\circ}$ and $142^{\circ}<\theta<168^{\circ}$.

The central tracking detector consists of a time expansion chamber (TEC) with high spatial resolution in the plane normal to the beam direction. TEC is surrounded by a Z-chamber which supplements the $r-\phi$ measurement with a $z$-coordinate measurement. The transverse momentum resolution of the tracking chamber is $\sigma\left(1 / P_{\mathrm{T}}\right)=0.018(\mathrm{GeV} / c)^{-1}$ in the central region $\left(\left|\cos \theta_{\mathrm{TEC}}\right|<0.7\right)$. The position resolution of a track extrapolated to the calorimeters is $0.2 \mathrm{~mm}$ in the plane transverse to the beam direction and $1.5 \mathrm{~mm}$ along the beam axis.

\section{Selection of $Z \rightarrow \tau^{+} \tau^{-}(\gamma)$ events}

The selection of $\tau$ decays is done independently in two hemispheres separated by the plane perpendicular to the thrust axis of the event. Particle identification in each hemisphere is based upon the topological properties of the energy deposition in the electromagnetic and hadron calorimeters with respect to the trajectory of the charged track.

The selection of $\tau$ decays proceeds in three steps. First, a sample of low multiplicity, back-to-back events is selected, consisting mainly of leptons from $\mathrm{Z}$ decays. This preselection suppresses such backgrounds as hadronic $\mathrm{Z}$ decays, two-photon interactions, cosmic muons, and beam-gas interactions. In the second step, the individual $\tau$ decays are identified. Finally, requirements are placed on the hemisphere opposite to the identified $\tau$ decay candidate in order to suppress the remaining non- $\tau$ background. The selection procedure is described in detail in Ref. [6]. A sample of 45262 events is selected in the fiducial volume $46^{\circ}<\theta<134^{\circ}$.

The selection efficiency for $Z \rightarrow \tau^{+} \tau^{-}(\gamma)$ events is calculated to be $57.8 \%$ using the KORALZ Monte Carlo program [7,8] with a full simulation of the L3 detector response ${ }^{5}$. The main source of inefficiency is the geometric acceptance. The $\mathrm{Z} \rightarrow \tau^{+} \tau^{-}(\gamma)$ Monte Carlo sample contains 674000 generated events, which corresponds to nine times more $\mathrm{Z} \rightarrow \tau^{+} \tau^{-}(\gamma)$ events than in the data sample. The background contamination from non- $\tau$ sources includes $1.9 \%$ of $\mathrm{e}^{+} \mathrm{e}^{-}(\gamma), 1.0 \%$ of $\mu^{+} \mu^{-}(\gamma), 0.4 \%$ of $\mathrm{Z} \rightarrow$ hadrons events, $0.4 \%$ of two-photon reactions, and $0.2 \%$ of cosmic muon events and beam-gas interactions. These estimates are derived primarily from the data using either the side opposite to the selected decay or global event characteristics. The corresponding Monte Carlo simulation for the background $[7,11]$ is used only to verify these estimates. The total number of $\mathrm{Z} \rightarrow \tau^{+} \tau^{-}(\gamma)$ events in the sample is estimated to be $43497 \pm 60$, where the error includes the uncertainty in the background evaluation.

\section{Selection of $\tau$ decays with neutral kaons}

To select a $\tau^{-} \rightarrow \nu_{\tau} \mathrm{K}^{0} \mathrm{X}^{-}$candidate ${ }^{6}$, exactly one track is required in the hemisphere. This track must be consistent with originating from the interaction point in the plane perpendicular to the beam direction. The expected energy deposition of the track in the HCAL is estimated from its momentum in the TEC and energy in the ECAL associated to it using the reconstruction technique described in Ref. [6]. Any HCAL energy deposition exceeding this estimate, $\Delta E_{\mathrm{HC}}=E_{\mathrm{HC}}^{\text {tot }}-$ $E_{\mathrm{HC}}^{\pi^{-}}>0$, could be due to a fluctuation in the charged hadron shower, which is approximately proportional to $\sqrt{P_{\pi}}$, or due to the presence of neutral kaons. In order to suppress fluctuations, the ratio $\Delta E_{\mathrm{HC}} / \sqrt{P_{\pi}}$ must be greater than $5(\mathrm{GeV})^{1 / 2}$ (Fig. 1a). This cut rejects a large fraction of $\tau$ decays with no $\mathrm{K}^{0}$ and almost all residual background from non- $\tau$ sources. No $\tau$ decays with the charged particle momentum greater than $15 \mathrm{GeV} / c$ survive this cut.

The dominant backgrounds after the previous cut are $\tau^{-} \rightarrow \rho^{-} \nu_{\tau}$ and $\tau^{-} \rightarrow \mathrm{a}_{1}^{-} \nu_{\tau}$ decays with $\pi^{0}$ leakage from the ECAL to the HCAL. This background is suppressed by requiring the energy in the first three

\footnotetext{
${ }^{5}$ The L3 detector simulation is based on GEANT Version 3.14; see Ref. [9]. The GHEISHA program [10] is used to simulate hadronic interactions.

${ }^{6}$ The hadronic system $\mathrm{X}^{-}$contains a single charged hadron and any number of neutral hadrons.
} 

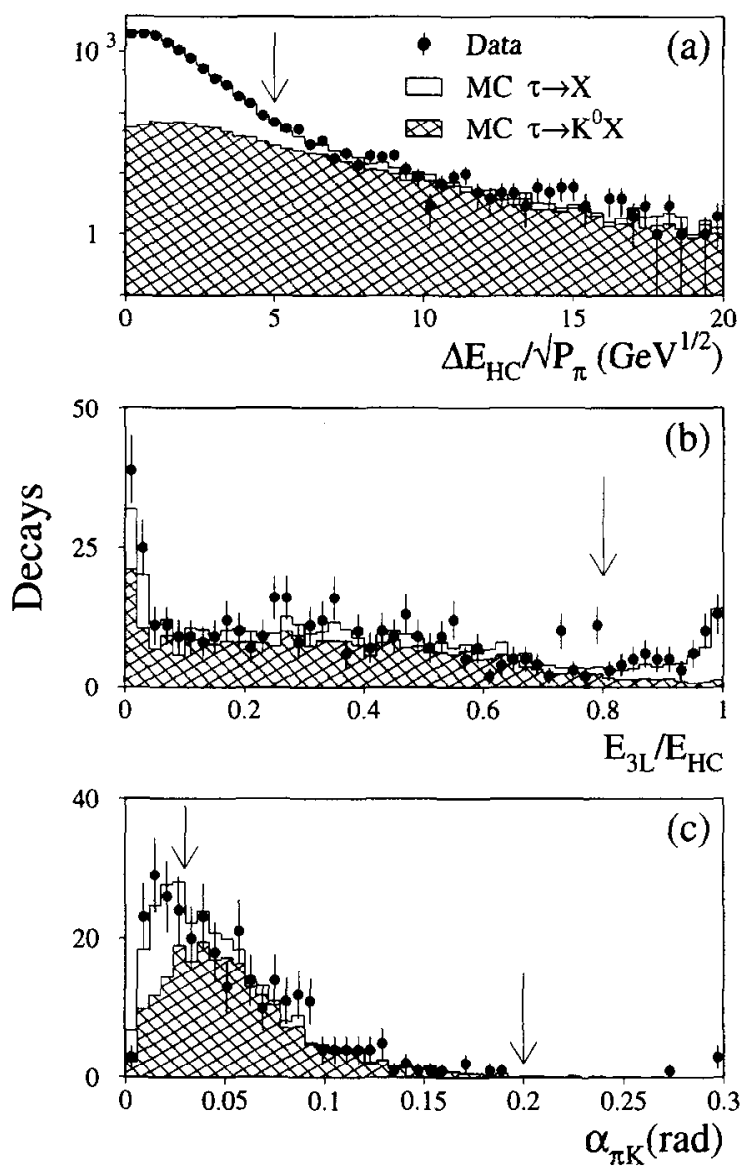

Fig. 1. (a) The spectrum of the ratio $\Delta E_{\mathrm{HC}} / \sqrt{P_{\pi}}$ for the preselected decays. The hatched area corresponds to the contribution of the $\tau^{-} \rightarrow \nu_{\tau} \pi^{-} \overline{\mathrm{K}}^{0}, \tau^{-} \rightarrow \nu_{\tau} \mathrm{K}^{-} \mathrm{K}^{0}, \tau^{-} \rightarrow \nu_{\tau} \pi^{-} \pi^{0} \mathrm{~K}^{0}$, $\tau^{-} \rightarrow \nu_{\tau} \pi^{-} \pi^{0} \overline{\mathrm{K}}^{0}, \tau^{-} \rightarrow \nu_{\tau} \mathrm{K}^{-} \pi^{0} \mathrm{~K}^{0}$ decay modes. (b) The spectrum of the fraction of the HCAL energy deposited in the first three layers. The cut on the ratio $\Delta E_{\mathrm{HC}} / \sqrt{P_{\pi}}$ is already applied. (c) The separation angle between the charged hadron and cluster in the HCAl. All other cuts are already applied. The arrows in (a)-(c) indicate the corresponding values of the applied cuts.

HCAL layers $\left(18 X_{0}\right)$ to be less than $80 \%$ of the total energy deposition in the HCAL (Fig. 1b). The angular separation between the $\pi^{-}$and $\mathrm{K}^{0}, \alpha_{\pi^{-}} \mathrm{K}^{0}$, is estimated as the difference between the center of gravity of the HCAL cluster and impact position of the track in the HCAL. It is required to be $0.03<\alpha_{\pi-K^{0}}<$ $0.2 \mathrm{rad}$ (Fig. 1c). This cut improves the purity of the selected sample.

After applying these selection criteria, $247 \tau^{-} \rightarrow$ $\nu_{r} \mathrm{~K}^{0} \mathrm{X}^{-}$candidate decays remain with 27.7 decays expected from the modes without $\mathbf{K}^{0}$ 's. The follow- ing $\tau$ decays contribute to the signal: $\tau^{-} \rightarrow \nu_{\tau} \pi^{-} \overline{\mathbf{K}}^{0}$ $\tau^{-} \rightarrow \nu_{\tau} \mathrm{K}^{-} \mathrm{K}^{0}, \tau^{-} \rightarrow \nu_{\tau} \pi^{-} \pi^{0} \mathrm{~K}^{0}, \tau^{-} \rightarrow \nu_{\tau} \mathrm{K}^{-} \pi^{0} \mathrm{~K}^{0}$ and $\tau^{-} \rightarrow \nu_{\tau} \pi^{-} \mathrm{K}^{0} \overline{\mathrm{K}}^{0}$. The efficiencies are estimated to be $13.0 \%, 5.3 \%, 8.8 \%, 3.7 \%$ and $19.8 \%$ in the fiducial volume respectively. The composition of the selected sample of $\mathrm{K}^{0}$ 's corresponds to $68 \%$ $\mathrm{K}_{\mathrm{L}}^{0}$ and $32 \% \mathrm{~K}_{\mathrm{S}}^{0}$. The modes $\tau^{-} \rightarrow \nu_{\tau} \mathrm{K}^{-} \mathrm{K}^{0}$ and $\tau^{-} \rightarrow \nu_{\tau} \mathrm{K}^{-} \pi^{0} \mathrm{~K}^{0}$ have significantly lower efficiency than the corresponding modes with charged pions due to less advantageous energy sharing between $\mathrm{K}^{-} \mathrm{K}^{0}$ in the laboratory frame compared to $\pi^{-} \overline{\mathrm{K}}^{07}$.

\section{Determination of the branching fractions}

In order to distinguish $\tau^{-} \rightarrow \nu_{\tau} \pi^{-} \overline{\mathrm{K}}^{0}$ decays from $\tau^{-} \rightarrow \nu_{\tau} \pi^{-} \pi^{0} \overline{\mathrm{K}}^{0}$ and $\tau^{-} \rightarrow \nu_{\tau} \pi^{-} \mathrm{K}^{0} \overline{\mathrm{K}}^{0}$ decays, the selected decays are classified into three categories:

(i) Decays with a $\pi^{0}$ candidate $\left(\tau^{-} \rightarrow \nu_{\tau} \pi^{-} \pi^{0} \overline{\mathrm{K}}^{0}\right.$ candidates).

These decays are required to have an energy cluster in the ECAL of greater than $6 \mathrm{GeV}$ and a transverse shower shape consistent with a $\pi^{0}$. A typical candidate is shown in Fig. 2.

(ii) Decays with extra neutral hadronic energy ( $\tau^{-} \rightarrow \nu_{\tau} \pi^{-} \mathrm{K}^{0} \overline{\mathrm{K}}^{0}$ candidates).

Two high energy hadronic showers from a $\mathrm{K}^{0} \overline{\mathrm{K}}^{0}$ pair are not separated in angle but are often separated in depth (Fig. 3). Therefore, they are characterized by a more uniform energy deposition than a single neutral hadronic shower. In order to select these decays, the energy deposition in the ECAL, and in the front and back parts of the HCAL are each required to exceed $10 \%$ of the total energy associated to neutral kaons.

(iii) Remaining decays ( $\tau^{-} \rightarrow \nu_{\tau} \pi^{-} \overline{\mathrm{K}}^{0}$ candidates). For each category, the total number of decays is a linear combination of the signal channels: $\tau^{-} \rightarrow \nu_{\tau} \pi^{-} \overline{\mathrm{K}}^{0}, \tau^{-} \rightarrow \nu_{\tau} \mathrm{K}^{-} \mathrm{K}^{0}, \tau^{-} \rightarrow \nu_{\tau} \pi^{-} \pi^{0} \overline{\mathrm{K}}^{0}$, $\tau^{-} \rightarrow \nu_{\tau} \mathrm{K}^{-} \pi^{0} \mathrm{~K}^{0}$, and $\tau^{-} \rightarrow \nu_{\tau} \pi^{-} \mathrm{K}^{0} \overline{\mathrm{K}}^{0}$ and the background channels. Only the coefficients for the modes $\tau^{-} \rightarrow \nu_{\tau} \pi^{-} \overline{\mathbf{K}}^{0}, \tau^{-} \rightarrow \nu_{\tau} \pi^{-} \pi^{0} \overline{\mathbf{K}}^{0}$, and $\tau^{-} \rightarrow \nu_{\tau} \pi^{-} \mathbf{K}^{0} \overline{\mathbf{K}}^{0}$ are allowed to vary in the fit.

\footnotetext{
${ }^{7}$ In the $\mathbf{K}-K^{0}$ final state the energy is on average divided equally between the charged and neutral kaons, leading to small values of the ratio $\Delta E_{\mathrm{HC}} / \sqrt{P_{\pi}}$. In the case of the $\pi^{-} \overline{\mathrm{K}}^{0}$ final state, the (heavy) neutral kaon has more energy in the laboratory frame than the (light) charged pion, leading to large values of $\Delta E_{\mathrm{HC}} / \sqrt{P_{\pi}}$.
} 


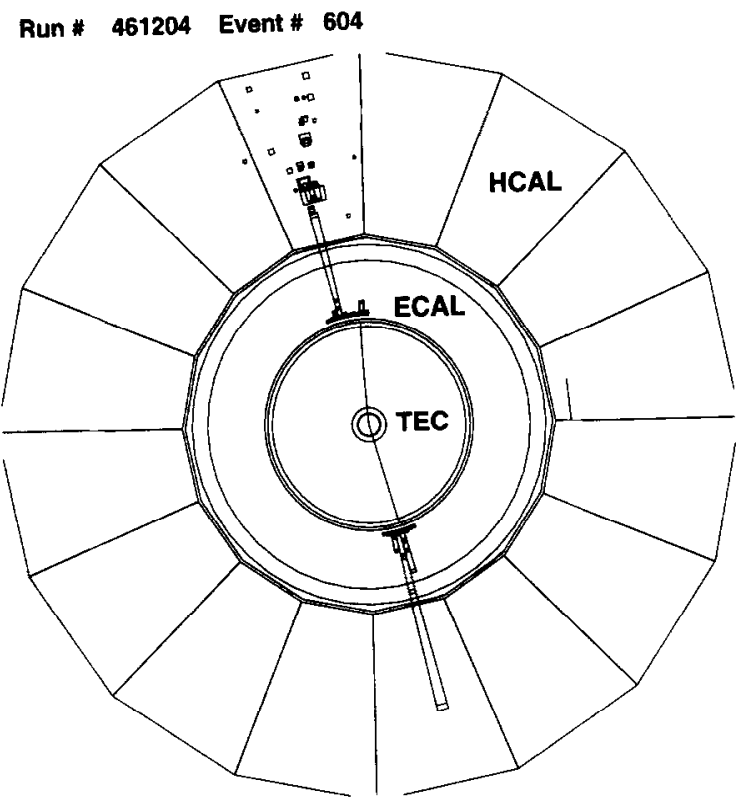

Fig. 2. A $\tau^{-} \rightarrow \nu_{\tau} \pi^{-} \pi^{0} \overline{\mathrm{K}}^{0}$ candidate decay. In the upper hemisphere, the $1.36 \mathrm{GeV} / c$ charged track is well separated in space from the $12.0 \mathrm{GeV} \pi^{0}$ detected in the ECAL and the $12.6 \mathrm{GeV}$ neutral hadron shower in the HCAL. The neutral hadron shower starts after $3 \mathrm{HCAL}$ layers (about $0.7 \lambda$ ).

The other coefficients are fixed to the central values from the recently published measurements [12]: $\mathcal{B}\left(\tau^{-} \rightarrow \nu_{\tau} \mathrm{K}^{-} \mathrm{K}^{0}\right)=0.0029 \pm 0.0012$ and $\mathcal{B}\left(\tau^{-} \rightarrow\right.$ $\left.\nu_{\tau} \mathrm{K}^{-} \pi^{0} \mathrm{~K}^{0}\right)=0.0005 \pm 0.0005$.

In order to increase the separation power between the channels, the coefficients of the linear combination are fit to a 10-bin histogram of the $\pi^{0}$ energy for the $\tau^{-} \rightarrow \nu_{r} \pi^{-} \pi^{0} \overline{\mathrm{K}}^{0}$ sample. A $5 \times 3$ binned histogram of total decay energy versus invariant mass of the observed $\tau$ decay products is used for the $\tau^{-} \rightarrow \nu_{\tau} \pi^{-} \overline{\mathbf{K}}^{0}$ and $\tau^{-} \rightarrow \nu_{\tau} \pi^{-} \mathbf{K}^{0} \overline{\mathbf{K}}^{0}$ samples. We use a binned maximum likelihood function which accounts for the finite statistics both in the data and in the Monte Carlo.

The spectrum used for the fit of the $\tau^{-} \rightarrow$ $\nu_{\tau} \pi^{-} \pi^{0} \overline{\mathrm{K}}^{0}$ sample is shown in Fig. 4a together with the best fit Monte Carlo distributions. The total energy and invariant mass distributions for the $\tau^{-} \rightarrow$ $\nu_{\tau} \pi^{-} \mathbf{K}^{0} \overline{\mathbf{K}}^{0}$ and $\tau^{-} \rightarrow \nu_{\tau} \pi^{-} \overline{\mathbf{K}}^{0}$ samples are shown in Figs. $4 \mathrm{~b}$ and $4 \mathrm{c}$ respectively together with the best fit Monte Carlo distributions.

The number of decays expected from the different channels is shown in Table 1 together with the corresponding number of data decays. The numbers of data
Run \# 509715 Event \# 2452

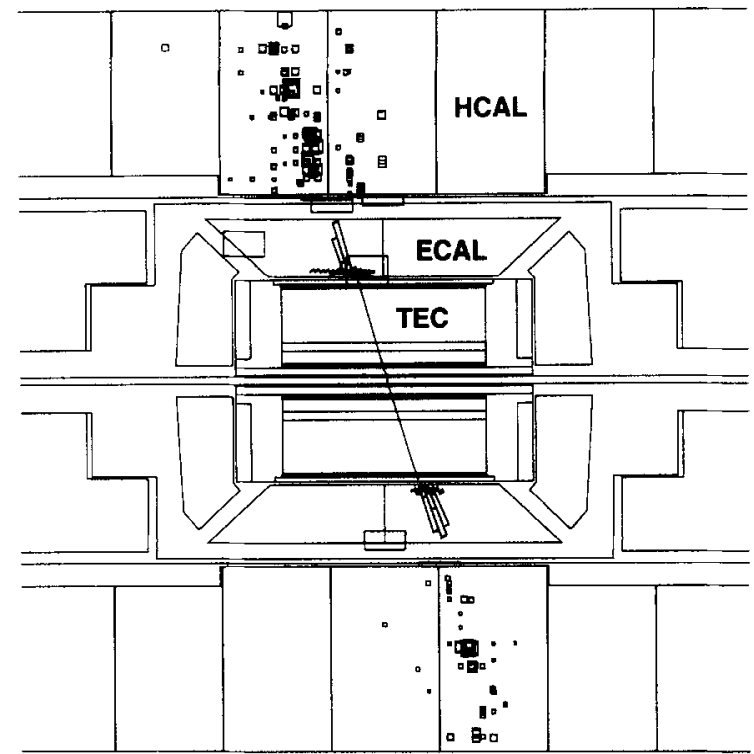

Fig. 3. A $\tau^{-} \rightarrow \nu_{\tau} \pi^{-} \mathbf{K}^{0} \overline{\mathbf{K}}^{0}$ candidate decay. In the upper hemisphere, the $2.39 \mathrm{GeV} / c$ charged track is separated in space from the $9.4 \mathrm{GeV}$ cluster in the ECAL and the $33.6 \mathrm{GeV}$ neutral hadron shower in the HCAL. The neutral hadron shower has two distinct local maxima: about $12 \mathrm{GeV}$ in the front part of the HCAL and $20 \mathrm{GeV}$ in the back part. The shower shape of the neutral energy cluster in the ECAL is inconsistent with being a $\pi^{0}$.

decays in each channel from the fit are

$$
\begin{aligned}
\mathrm{N}\left(\tau^{-}, \nu_{\tau} \pi^{-} \overline{\mathbf{K}}^{0}\right) & =112.0 \pm 17.7 \\
\mathrm{~N}\left(\tau^{-} \rightarrow \nu_{\tau} \pi^{-} \pi^{0} \overline{\mathrm{K}}^{0}\right) & =33.1 \pm 9.7 \\
\mathrm{~N}\left(\tau^{-} \rightarrow \nu_{\tau} \pi^{-} \mathrm{K}^{0} \overline{\mathrm{K}}^{0}\right) & =55.8 \pm 21.4
\end{aligned}
$$

and the correlation coefficients between the fitted channels are

$$
\begin{array}{r}
\rho\left(\pi^{-} \overline{\mathrm{K}}^{0}, \pi^{-} \pi^{0} \overline{\mathrm{K}}^{0}\right)=0.01 \\
\rho\left(\pi^{-} \overline{\mathrm{K}}^{0}, \pi^{-} \mathrm{K}^{0} \overline{\mathrm{K}}^{0}\right)=0.68 \\
\rho\left(\pi^{-} \pi^{0} \overline{\mathrm{K}}^{0}, \pi^{-} \mathrm{K}^{0} \overline{\mathrm{K}}^{0}\right)=0.36 .
\end{array}
$$

The large correlation coefficient between $\mathrm{N}\left(\tau^{-} \rightarrow\right.$ $\left.\nu_{\tau} \pi^{-} \overline{\mathrm{K}}^{0}\right)$ and $\mathrm{N}\left(\tau^{-} \rightarrow \nu_{\tau} \pi^{-} \mathrm{K}^{0} \overline{\mathrm{K}}^{0}\right)$ results in an increase of the statistical errors for the corresponding branching fractions. If we fix $\mathrm{N}\left(\tau^{-} \rightarrow \nu_{\tau} \pi^{-} \mathrm{K}^{0} \overline{\mathrm{K}}^{0}\right)$ in the fit, the corresponding error for $\mathrm{N}\left(\tau^{-} \rightarrow \nu_{\tau} \pi^{-} \overline{\mathbf{K}}^{0}\right)$ is significantly reduced: $\mathrm{N}\left(\tau^{-} \rightarrow \nu_{\tau} \pi^{-} \overline{\mathbf{K}}^{0}\right)=112.0 \pm$ 12.8. Therefore, it is important to account for this correlation. 

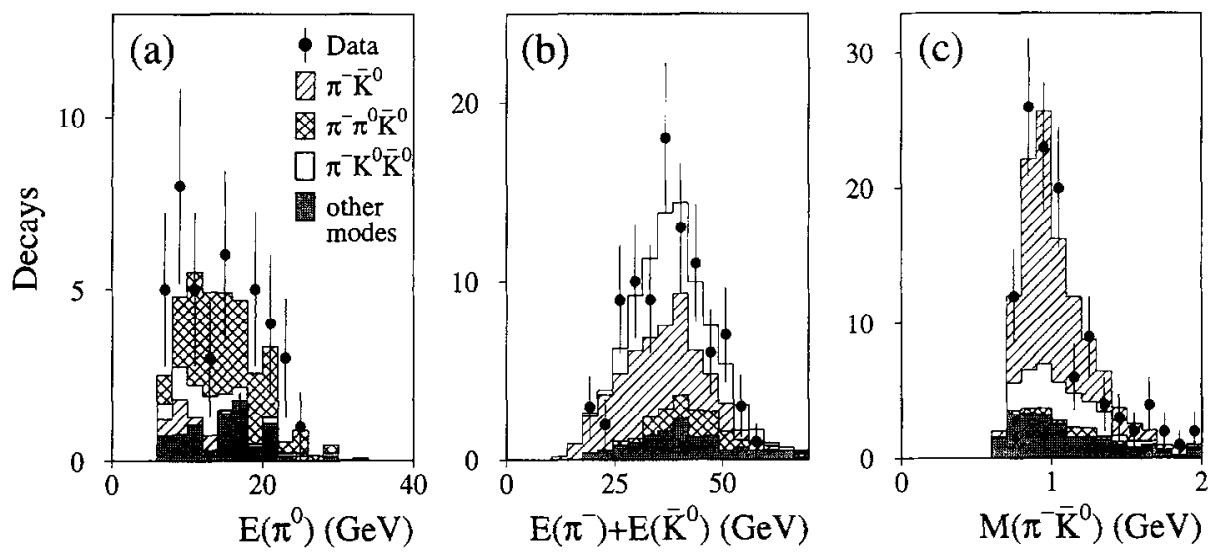

Fig. 4. (a) The spectrum of the $\pi^{0}$ energy for the sample enriched with $\tau^{-} \rightarrow \nu_{\tau} \pi^{-} \pi^{0} \bar{K}^{0}$ decays. (b) The spectrum of the sum of energies of the observed $\tau$ decay products for the sample enriched with $\tau^{-} \rightarrow \nu_{\tau} \pi^{-} \mathbf{K}^{0} \overline{\mathrm{K}}^{0}$ decays. (c) The invariant mass spectrum of the $\pi^{-} \overline{\mathrm{K}}^{0}$ system for the sample enriched with $\tau^{-} \rightarrow \nu_{\tau} \pi^{-} \overline{\mathbf{K}}^{0}$ decays.

Table 1

The numbers of decays detected in the three subsamples along with the best fit Monte Carlo expectations. For the $\tau^{-} \rightarrow \nu_{\tau} \mathrm{K}^{-} \mathrm{K}^{0}$ and $\tau^{-} \rightarrow \nu_{\tau} \mathrm{K}^{-} \pi^{0} \mathrm{~K}^{0}$ channels, the numbers of expected decays correspond to the branching fractions measured in Ref. [12]

\begin{tabular}{|c|c|c|c|c|}
\hline & $\pi^{-} \overrightarrow{\mathbf{K}}^{0}$ sample & $\pi^{-} \pi^{0} \overline{\mathbf{K}}^{0}$ sample & $\pi^{-} \mathrm{K}^{0} \overline{\mathrm{K}}^{0}$ sample & Total \\
\hline Data & 114 & 41 & 92 & 247 \\
\hline$\tau^{-} \rightarrow \nu_{\tau}^{\prime} \pi^{-} \tilde{\mathrm{K}}^{0}$ & 71.9 & 2.4 & 37.7 & 112.0 \\
\hline$\tau^{-} \rightarrow \nu_{\tau} K^{-} \mathbf{K}^{0}$ & 10.0 & 0.1 & 3.8 & 13.9 \\
\hline$\tau^{-} \rightarrow \nu_{\tau} \pi^{-} \pi^{0} \overline{\mathbf{K}}^{0}$ & 3.5 & 20.3 & 9.3 & 33.1 \\
\hline$\tau \rightarrow \nu_{\tau} K^{-} \pi^{0} \mathbf{K}^{0}$ & 0.4 & 0.3 & 1.0 & 1.7 \\
\hline$\tau^{-} \rightarrow \nu_{\tau} \pi^{-} \mathrm{K}^{0} \overline{\mathrm{K}}^{0}$ & 18.9 & 4.9 & 32.0 & 55.8 \\
\hline Background & 11.8 & 7.5 & 8.4 & 27.7 \\
\hline MC total & 116.5 & 35.5 & 82.2 & 244.2 \\
\hline
\end{tabular}

\section{Systematic uncertainties}

The dominant systematic errors in the measurement arise from the uncertainties in the absolute energy calibration and detector resolution functions, the background estimation, and theoretical uncertainties.

The calibration uncertainties affect both the selection efficiency and the shape of the final distributions used for the fit. The accuracy of the ECAL energy scale for electrons and photons is estimated to be $1 \%$ at $1 \mathrm{GeV}$ from the measurement of the position of the $\pi^{0}$ mass peak and $0.1 \%$ at $45 \mathrm{GeV}$ from a study of $\mathrm{Z} \rightarrow \mathrm{e}^{+} \mathrm{e}^{-}(\gamma)$ events. The momentum scale of the central tracker is verified to $1 \%$ accuracy from 1 to $45 \mathrm{GeV}$ using low energy electrons as well as muons from $\tau$ and $Z$ decays. The absolute energy scales of the ECAL and HCAL for hadrons are each known to $1.5 \%$ in the central region from the position of the $\rho$ mass peak. The corresponding uncertainties in the number of signal decays propagated through the selection and fitting procedures are listed in Table 2 .

The resolution function of the HCAL is verified using test beam data and $\tau^{-} \rightarrow \rho^{-} \nu_{\tau}$ decays. The spectra of the ratio $\Delta E_{\mathrm{HC}} / \sqrt{P_{\pi}}$, as measured in the test beam for single pions of different energies, are compared with the Monte Carlo expectations in Fig. 5. No discrepancy between data and Monte Carlo is observed within the achieved statistical errors, limiting possible uncertainty of the non- $\mathrm{K}^{0}$ background to \pm 2.1 decays. The uncertainty in the number of decays with 
Table 2

Summary of the systematic uncertainties in the numbers of fitted decays

\begin{tabular}{|c|c|c|c|c|}
\hline Source & & $\Delta \mathbf{N}_{\pi}-\bar{k}^{0}$ & $\Delta \mathbf{N}_{\pi^{-}-\pi^{0} \mathbb{K}^{0}}$ & $\Delta \mathbf{N}_{\pi}-\mathbf{K}^{0} \overline{\mathbf{K}}^{0}$ \\
\hline \multirow[t]{3}{*}{ Calibration } & HCAL & 3.5 & 0.9 & 4.3 \\
\hline & ECAL & 1.0 & 1.6 & 2.5 \\
\hline & TEC & 1.0 & 0.8 & 0.6 \\
\hline \multirow[t]{4}{*}{ Background } & $\pi^{0}$ & 2.0 & 0.8 & 0.6 \\
\hline & $\pi^{ \pm} / \mathrm{K}^{ \pm}$ & 1.5 & 0.5 & 0.6 \\
\hline & $\Delta \mathrm{N}_{\mathrm{K}}-\mathrm{K}^{0}$ & 3.4 & 0.2 & 2.4 \\
\hline & $\Delta \mathrm{N}_{\mathrm{K}}-\pi^{0} \mathrm{~K}^{0}$ & 0.2 & 0.8 & 0.9 \\
\hline \multirow[t]{2}{*}{ Theory } & matrix element & 3.9 & 1.2 & 4.0 \\
\hline & $\mathrm{CP}\left(\mathrm{K}^{0} \overline{\mathbf{K}}^{0}\right)$ & - & - & 2.7 \\
\hline Total & $\Delta \mathrm{N}^{\text {syst }}$ & 6.9 & 2.6 & 7.4 \\
\hline
\end{tabular}

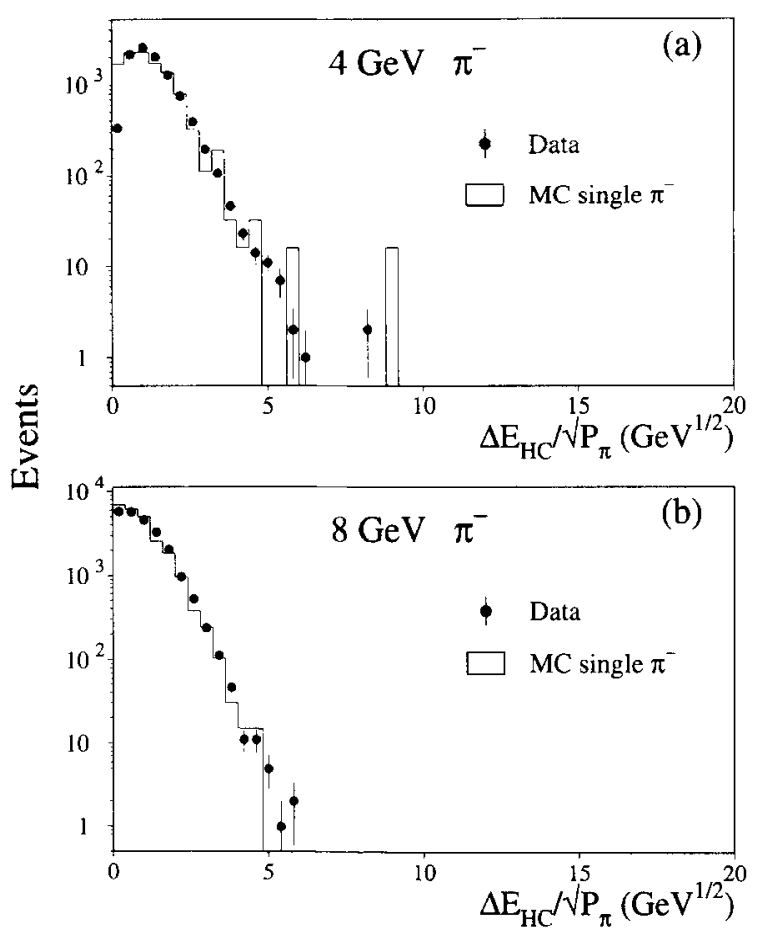

Fig. 5. The spectra of the ratio $\Delta E_{\mathrm{HC}} / \sqrt{P_{\pi}}$ for (a) $4 \mathrm{GeV}$ and (b) $8 \mathrm{GeV}$ single pions as measured in the test-beam (dots) and predicted by the Monte Carlo (solid histogram).

$\mathrm{K}^{0}$ 's due to inefficiency of the HCAL is estimated to be $1 \%$ of the number of fitted decays from a study of $\mathrm{Z} \rightarrow \mu^{+} \mu^{-}(\gamma)$ events.

The systematic error due to the uncertainty in the rate of the decays $\tau^{-} \rightarrow \nu_{\tau} \mathrm{K}^{-} \mathrm{K}^{0}$ and $\tau^{-} \rightarrow$ $\nu_{\tau} \mathrm{K}^{-} \pi^{0} \mathrm{~K}^{0}$ is estimated by varying their branching fractions according to the uncertainties quoted in Ref. [12]. Uncertainties in the number of $\pi^{0}$ 's from $\tau^{-} \rightarrow \rho^{-} \nu_{\tau}$ and $\tau^{-} \rightarrow a_{1}^{-} \nu_{\tau}$ decays leaking to the HCAL are estimated using a sample enriched with $\pi^{0}$ 's with abnormally high energy deposition in the first three layers of the HCAL (seen as a peak at $E_{3 \mathrm{~L}} / E_{\mathrm{HC}} \approx 1$ in Fig. $1 \mathrm{~b}$ ). This uncertainty is estimated to be $20 \%$ of the number of $\tau^{-} \rightarrow \rho^{-} \nu_{\tau}$ and $\tau^{-} \rightarrow \mathrm{a}_{1}^{-} \nu_{\tau}$ decays predicted by Monte Carlo simulation.

The theoretical error due to the uncertainty in the matrix element for the $\tau$ decays into three particles with one or two kaons arises from the non-uniformity of the selection efficiency over the phase space. In the Monte Carlo program [8] used to simulate $\tau$ decays, assumptions are made about the chiral structure of the weak hadronic current and the resonant structure of these decays. By varying these assumptions, we estimate the possible change of the efficiencies to be of the order $10 \%$, depending slightly on the decay channel; the exact numbers are listed in Table 2. Another source of systematic error is the uncertainty in the CP phase of the $\mathrm{K}^{0} \overline{\mathrm{K}}^{0}$ system in the decay $\tau^{-} \rightarrow$ $\nu_{\tau} \pi^{-} \mathrm{K}^{0} \overline{\mathrm{K}}^{0}$. The systematic error is assigned according to the difference in the fit results assuming that the $\mathrm{K}^{0} \overline{\mathrm{K}}^{0}$ sample consists of only $\mathrm{CP}$-odd or only $\mathrm{CP}$ even components. In the final fit, used to derive the branching fractions, the following proportion is assumed $\mathrm{K}_{\mathrm{L}} \mathrm{K}_{\mathrm{L}}: \mathrm{K}_{\mathrm{S}} \mathrm{K}_{\mathrm{S}}: \mathrm{K}_{\mathrm{S}} \mathrm{K}_{\mathrm{L}}=0.25: 0.25: 0.5$.

Table 2 summarizes the study of the systematic errors for the numbers of fitted decays. When combining the systematic errors of a given channel, all sources are assumed to be independent.

\section{Discussion and conclusions}

From the fit results presented in Table 1, the selection efficiencies, and the total number of observed $\tau$ decays, we extract the following branching fractions:

$$
\begin{aligned}
& \mathcal{B}\left(\tau^{-} \rightarrow \nu_{\tau} \pi^{-} \overline{\mathrm{K}}^{0}\right)=0.0095 \pm 0.0015 \text { (stat) } \\
& \quad \pm 0.0006 \text { (syst) } \\
& \mathcal{B}\left(\tau^{-} \rightarrow \nu_{\tau} \pi^{-} \pi^{0} \overline{\mathrm{K}}^{0}\right)=0.0041 \pm 0.0012 \text { (stat) } \\
& \quad \pm 0.0003 \text { (syst) }
\end{aligned}
$$



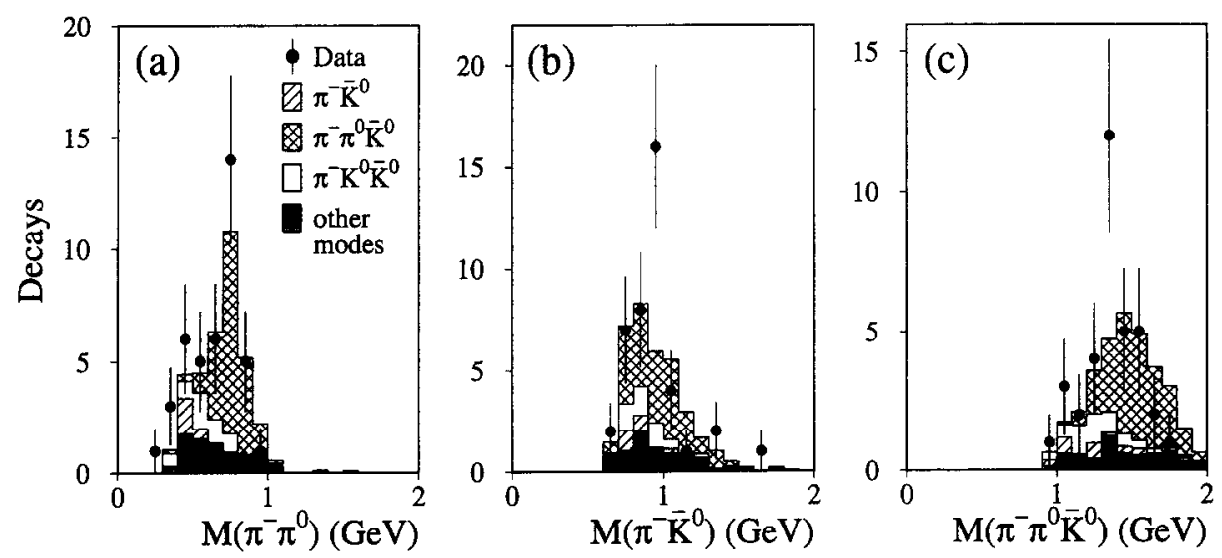

Fig. 6. (a) The invariant mass spectrum of the $\pi^{-} \pi^{0}$ system for the sample enriched with $\tau^{-} \rightarrow \nu_{\tau} \pi^{-} \pi^{0} \overline{\mathrm{K}}^{0}$ decays. (b) The invariant mass spectrum of the $\pi^{-} \overline{\mathrm{K}}^{0}$ system for the sample enriched with $\tau^{-} \rightarrow \nu_{\tau} \pi^{-} \pi^{0} \overline{\mathrm{K}}^{0}$ decays. (c) The invariant mass spectrum of the $\pi^{-} \pi^{0} \overline{\mathrm{K}}^{0}$ system for the sample enriched with $\tau^{-} \rightarrow \nu_{\tau} \pi^{-} \pi^{0} \overline{\mathrm{K}}^{0}$ decays.

$$
\begin{aligned}
& \mathcal{B}\left(\tau^{-} \rightarrow \nu_{\tau} \pi^{-} \mathrm{K}^{0} \overline{\mathrm{K}}^{0}\right)=0.0031 \pm 0.0012 \text { (stat) } \\
& \quad \pm 0.0004 \text { (syst) }
\end{aligned}
$$

These results are consistent with other recent measurements [12-15] and comparable in accuracy.

Despite the absence of explicit identification of the final state charged hadron, these measurements are largely independent of the assumed branching fractions for $\tau^{-} \rightarrow \nu_{\tau} \mathrm{K}^{-} \mathrm{K}^{0}$ and $\tau^{-} \rightarrow \nu_{\tau} \mathrm{K}^{-} \pi^{0} \mathrm{~K}^{0}$. This is due to the fact that the selection efficiencies for these modes are significantly lower than the corresponding efficiencies for modes with charged pions, $\tau^{-} \rightarrow \nu_{\tau} \pi^{-} \overline{\mathbf{K}}^{0}$ and $\tau^{-} \rightarrow \nu_{\tau} \pi^{-} \pi^{0} \overline{\mathbf{K}}^{0}$, as a result of the different kinematics of these decays.

The invariant mass spectrum of the $\pi^{-} \overline{\mathrm{K}}^{0}$ system agrees with the model that this final state comes only from $\tau^{-} \rightarrow \nu_{\tau} \mathrm{K}^{*-} \rightarrow \nu_{\tau} \pi^{-} \overline{\mathbf{K}}^{0}$. Assuming no other contributions to this final state, we extract

$$
\begin{aligned}
& \mathcal{B}\left(\tau^{-} \rightarrow \nu_{\tau} \mathrm{K}^{*-}\right)=0.0142 \pm 0.0022 \text { (stat) } \\
& \quad \pm 0.009 \text { (syst) }
\end{aligned}
$$

We have also considered the production of the $\pi^{-} \overline{\mathrm{K}}^{0}$ final state from higher mass resonances or nonresonant $\tau^{-}$decays. Including these modes in the fit of our data, the following limits are derived:

$$
\begin{aligned}
& \mathcal{B}\left(\tau^{-} \rightarrow \nu_{\tau} \pi^{-} \overline{\mathbf{K}}^{0}\right)_{\text {nonresonant }}<0.0017 \text { at } 95 \% \mathrm{CL} \\
& \mathcal{B}\left(\tau^{-} \rightarrow \nu_{\tau} \pi^{-} \overline{\mathbf{K}}^{0}\right)_{\mathrm{K}^{*-}(1430)}<0.0011 \text { at } 95 \% \mathrm{CL}
\end{aligned}
$$

The latter does not depend on the assumption of the spin of the $\mathrm{K}^{*-}(1430)$ resonance.

We have studied the resonant structure of the hadronic current in the $\tau^{-} \rightarrow \nu_{\tau} \pi^{-} \pi^{0} \overline{\mathrm{K}}^{0}$ decay (Fig. 6). The $\rho^{-}$peak in the $\pi^{-} \pi^{0}$ invariant mass distribution is well reproduced by the Monte Carlo model [8] as shown in Fig. 6a. However, Fig. 6b and Fig. $6 \mathrm{c}$ indicate a possible enhancement of the decays $\tau^{-} \rightarrow \nu_{\tau} \mathrm{K}_{1}^{*-} \rightarrow \nu_{\tau} \mathrm{K}^{*} \pi \rightarrow \nu_{\tau} \pi^{-} \pi^{0} \overline{\mathrm{K}}^{0}$ in the data.

Our measurement of the branching fraction $\mathcal{B}\left(\tau^{-} \rightarrow \nu_{\tau} \pi^{-} \mathrm{K}^{0} \overline{\mathrm{K}}^{0}\right)$ is insensitive to the theoretical assumptions on the matrix element and the $\mathrm{CP}$ phase of the $\mathrm{K}^{0} \overline{\mathrm{K}}^{0}$ system. It is consistent within the achieved accuracy with measurements of the similar decay $\tau^{-} \rightarrow \nu_{\tau} \pi^{-} \mathrm{K}^{-} \mathrm{K}^{+}[13,15]$.

\section{Acknowledgments}

We wish to express our gratitude to the CERN accelerator divisions for the excellent performance of the LEP machine. We acknowledge the efforts of all engineers and technicians who have participated in the construction and maintenance of this experiment.

\section{References}

[1] Particle Data Group, L. Montanet et al., Review of Particle Properties, Phys. Rev. D 50 (1994) 1406.

[2] R. Decker et al., Phys. Rev. D 50 (1994) 6863. 
[3] E. Braaten et al., Int. J. Mod. Phys. A 5 (1990) 2737.

[4] L3 Collaboration, B. Adeva et al., Nucl. Instr. and Meth. A 289 (1990) 35;

O. Adriani et al., Nucl. Instr. and Meth. A 302 (1991) 53; K. Deiters et al., Nucl. Instr. and Meth. A 323 (1992) 162; J. A. Bakken, Nucl. Instr. and Meth. A 275 (1989) 81.

[5] A. Arefiev et al., Nucl. Instr. and Meth. A 251 (1986) 258; A. Arefiev et al., Nucl. Instr. and Meth. A 285 (1989) 403.

[6] L3 Collaboration, O.Adriani et al., Phys. Lett. B 294 (1992) 466: B 341 (1994) 245.

[7] S. Jadach, B.F.L. Ward and Z. Wags, Comp. Phys. Comm. 66 (1991) 276;

$R$. Kleiss et al., in " $Z$ Physics at LEP1", CERN Report CERN 89-08 (1989), Vol. 3, p. 1.

[8] S. Jadach et al., Comp. Phys. Comm. 76 (1994) 361;

S. Jadach, J.H. Kühn and Z. Wạs, Comp. Phys. Comm. 64 (1991) 275
[9] R. Brun et al., "GEANT 3", CERN DD/EE/84-1 (Revised), September 1987.

[10] H. Fesefeldt, RWTH Aachen Preprint PITHA 85/02 (1985).

[11] T. Sjöstrand and M. Bengtsson, Comp. Phys. Comm. 43 (1987) 367;

T. Sjöstrand et al., in " $Z$ Physics at LEP1", CERN Report CERN 89-08 (1989), Vol. 3, p. 143.

[12] ALEPH Collaboration, D. Buskulic et al., Phys. Lett. B 332 (1994) 219.

[13] DELCO Collaboration, G.B. Mills et al., Phys. Rev. Lett. 54 (1985) 624.

[14] OPAL Collaboration, R. Akers et al., Phys. Lett. B 339 (1994) 278.

[15] TPC $/ 2 \gamma$ Collaboration, D.A. Bauer et al., Phys. Rev. D 50 (1994) 13. 